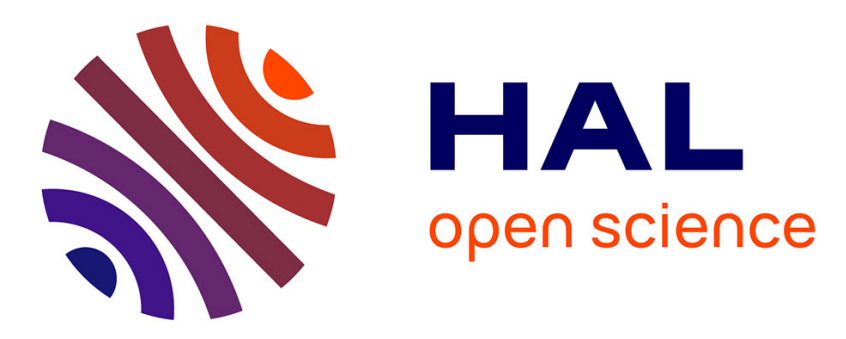

\title{
Pseudo-conforming polynomial finite elements on quadrilaterals
}

\author{
Eric Dubach, Robert Luce, Jean-Marie Thomas
}

\section{To cite this version:}

Eric Dubach, Robert Luce, Jean-Marie Thomas. Pseudo-conforming polynomial finite elements on quadrilaterals. International Journal of Computer Mathematics, 2009, 86 (10-11), pp.1798-1816. 10.1080/00207160902759342 . inria-00342656

\section{HAL Id: inria-00342656 \\ https://hal.inria.fr/inria-00342656}

Submitted on 27 Nov 2008

HAL is a multi-disciplinary open access archive for the deposit and dissemination of scientific research documents, whether they are published or not. The documents may come from teaching and research institutions in France or abroad, or from public or private research centers.
L'archive ouverte pluridisciplinaire HAL, est destinée au dépôt et à la diffusion de documents scientifiques de niveau recherche, publiés ou non, émanant des établissements d'enseignement et de recherche français ou étrangers, des laboratoires publics ou privés. 


\title{
RESEARCH ARTICLE
}

\section{Pseudo-conforming polynomial finite elements on quadrilaterals}

\author{
Eric DUBACH*, Robert LUCE*,†, Jean-Marie THOMAS* \\ (Received 00 Month 200x; in final form 00 Month 200x)
}

\begin{abstract}
The aim of this paper is to present a new class of finite elements on quadrilaterals where the approximation is polynomial on each element $K$. In the case of Lagrange finite elements, the degrees of freedom are the values at the vertices and in the case of mixed finite elements the degrees of freedom are the mean values of the fluxes on each side. The degres of freedom are the same as those of classical finite elements. However, in general, with this kind of finite elements, the resolution of second order elliptic problems leads to non conforming approximations. In the particular case when the finite elements are parallelograms, we can notice that our method is conform and coincides with the classical finite elements on structured meshes.

First, a motivation for the study of the Pseudo-conforming polynomial finite elements method is given, and the convergence of the method established. Then, numerical results that confirm the error estimates, predicted by the theory, are presented.
\end{abstract}

Keywords: Lagrange and mixed finite elements, polynomial approximation, non conforming approximation, quadrilateral meshes

AMS Subject Classification: 65N15, 65N15, 65N30

\section{Introduction}

Quadrilaterals and hexahedra are often used in meshers particularly in geophysical applications and in fluids mechanics. When the geometry and the medium are structured, regular rectangular meshes are used. Otherwise general convex quadrilaterals or hexahedra (or bricks) are used. Then, with isoparametric Lagrange finite elements $([1],[2],[6])$ or mixed finite elements $([3],[5])$, we must construct finite elements on the mesh by using multilinear mappings noted $F$ to a reference rectangle or rectangular solid.

For Lagrange isoparametric finite elements the jacobian of these mappings leads to non polynomial basis functions on the elements of the mesh and introduces non polynomial matrices in the partial differential operators. For mixed finite elements, consequences are even worse since the use of the Piola transform to work on the reference element is effective only when the mapping is linear otherwise a loss of order of convergence is observed ([7]).

In this paper, we are interested in quadrilateral meshes. To built our finite elements, we consider a quadrilateral as a distortion of a parallelogram and the Lagrange basis functions are built under conditions of weak-continuity of the unknowns accross the elements. The obtained finite elements are not conform but the conditions of weak-continuity are sufficient to ensure the expected order of convergence. Finite elements of lower degree are presented. We focus on the process to obtain the finite elements and we present some thereotical and technical results.

We use the following notations:

*Laboratoire de Mathématiques Appliquées, UMR 5142, Université de Pau et des Pays de l'Adour, BP 1155, 64013 Pau Cedex, France.

$\dagger$ INRIA Sud-Ouest

ISSN: 0020-7160 print/ISSN 1029-0265 online

(C) 200x Taylor \& Francis

DOI: $10.1080 / 0020716 Y Y x x x x x x x x$

http://www.informaworld.com 
For a vector $\mathbf{v} \in \mathbb{R}^{\mathbf{n}}, v=\left(v_{1}, \ldots, v_{n}\right)$ and $|\mathbf{v}|=\left\{\sum_{j=1}^{n}\left|v_{j}\right|^{2}\right\}^{1 / 2}$. For a square matrix $B,\|B\|$ is the spectral norm.

For a triangle or a quadrilateral $K,|K|$ denotes the area of $K$, and $|\gamma|$ the length of an edge $\gamma$ of $K$.

For a polyhedral domain $K$, we define

$$
H^{m}(K)=\left\{v \in L^{2}(K) ; \partial^{\alpha} v \in L^{2}(K), \text { for all } \alpha \text { with }|\alpha| \leq m\right\}
$$

equipped with the norm and the semi-norm

$$
\|v\|_{m, K}=\left(\sum_{|\alpha| \leq m} \int_{K}\left|\partial^{\alpha} v\right|^{2} d x\right)^{1 / 2}, \quad|v|_{m, K}=\left(\sum_{|\alpha|=m} \int_{K}\left|\partial^{\alpha} v\right|^{2} d x\right)^{1 / 2} .
$$

We consider also the following norm and semi-norm

$$
\|v\|_{m, \infty, K}=\max _{|\alpha| \leq m}\left\{\operatorname{ess}_{x \in K}\left|\partial^{\alpha} v\right|\right\}, \quad|v|_{m, \infty, K}=\max _{|\alpha|=m}\left\{\operatorname{ess} \sup _{x \in K}\left|\partial^{\alpha} v\right|\right\} .
$$

Equally, we define

$$
H(\operatorname{div}, K)=\left\{\mathbf{q} \in L^{2}(K) \times L^{2}(K) ; \operatorname{div}(\mathbf{q}) \in L^{2}(K)\right\}
$$

equipped with the norm

$$
\|\mathbf{q}\|_{H \operatorname{div}(0, K)}=\left(\int_{K}|\mathbf{q}|^{2} d x+\int_{K}|\operatorname{div}(\mathbf{q})|^{2} d x\right)^{1 / 2} .
$$

$\mathbf{q} \in H^{m}(K)$ means that all the component of $\mathbf{q}$ are in $H^{m}(K)$. Let $P(K)$ be the vectorial space $\{\mathbf{x} \in K \mapsto p(\mathbf{x}) ; p \in P\}$, where $P$ is a $N$ variables polynomial space and $K$ is a domain in $\mathbb{R}^{N}$. For any integer $k, P_{k}$ denotes the space of polynomial functions of degree $\leq k$, while $Q_{k}$ is the space of polyomial functions of degree $\leq k$ in each variable.

For each polyhedral $K, h_{K}$ denotes the diameter of $K$ and $\rho_{K}$ denotes the diameter of the largest ball contained in $K$.

\section{The finite element geometry.}

\subsection{The geometry; vertex and face numbering.}

Let $K$ be a convex nondegenerated quadrilateral. Let $\left\{\mathbf{a}_{i} \in \mathbb{R}^{2}, 1 \leq i \leq 4\right\}$ be the vertices of $K$.

Two vertices which do not belong to the same edge of $K$ are said to be opposite vertices. The center of a polyhedral is the isobarycenter of its vertices; we denote by $\mathbf{a}_{0}$ the center of $K$ :

$$
\mathbf{a}_{0}=\frac{1}{4} \sum_{1 \leq i \leq 4} \mathbf{a}_{i}
$$

Let now $\left\{\gamma_{m}, 1 \leq m \leq 4\right\}$ be the set of the edges of $K$. Two edges without common vertex are said opposite edges. The vertex and face numbering is shown on Figure 

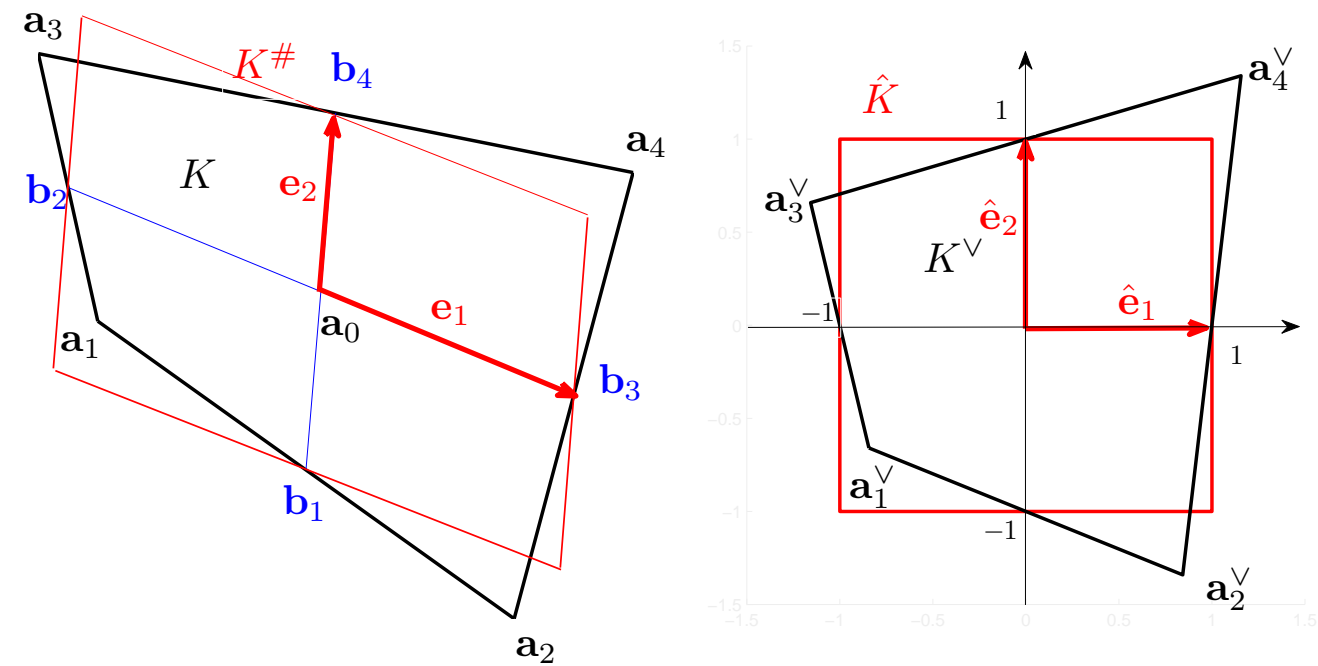

Figure 1. Numerotation

1. This numbering is such that:

$$
\begin{aligned}
& \gamma_{1}=\left(\mathbf{a}_{1}, \mathbf{a}_{2}\right), \gamma_{2}=\left(\mathbf{a}_{1}, \mathbf{a}_{3}\right), \\
& a_{5-m} \text { opposite } a_{m}, \text { and } \\
& \gamma_{5-m} \text { opposite } \gamma_{m} ; \text { for } m=1,2 .
\end{aligned}
$$

Last, let $\mathbf{b}_{m}$ be the center of the face $\gamma_{m}$, for $m=1, \ldots, 4$, and let us introduce the

Last, let $\mathbf{b}_{m}$ be the center of the face $\gamma_{m}$, for $m=1, \ldots, 4$, and let us introduce the
vectors $\mathbf{e}_{1}, \mathbf{e}_{2} \in \mathbb{R}^{2}$ defined by

$$
\mathbf{e}_{1}=\mathbf{a}_{0}-\mathbf{b}_{2}=\mathbf{b}_{3}-\mathbf{a}_{0}, \quad \mathbf{e}_{2}=\mathbf{a}_{0}-\mathbf{b}_{1}=\mathbf{b}_{4}-\mathbf{a}_{0}
$$

Since $K$ is assumed to be a nondegenerated quadrilateral, $\left(\mathbf{e}_{1}, \mathbf{e}_{2}\right)$ is a basis of $\mathbb{R}^{2}$.

\title{
2.2 Affine-equivalent elements.
} We choose

$$
\widehat{\mathbf{a}}_{1}=\left(\begin{array}{l}
-1 \\
-1
\end{array}\right), \widehat{\mathbf{a}}_{2}=\left(\begin{array}{l}
+1 \\
-1
\end{array}\right)
$$

and the vertex and face numbering is made as previously. Let $\widehat{\mathbf{b}}_{m}$ be the center
of the edge $\widehat{\gamma}_{m}$, for $m=1, \ldots, 4$. The canonical basis $\left(\widehat{\mathbf{e}}_{1}, \widehat{\mathbf{e}}_{2}\right)$ of $\mathbb{R}^{2}$ can be simply
expressed with the vectors $\widehat{\mathbf{b}}_{m}$

and the vertex and face numbering is made as previously. Let $\widehat{\mathbf{b}}_{m}$ be the center
of the edge $\widehat{\gamma}_{m}$, for $m=1, \ldots, 4$. The canonical basis $\left(\widehat{\mathbf{e}}_{1}, \widehat{\mathbf{e}}_{2}\right)$ of $\mathbb{R}^{2}$ can be simply
expressed with the vectors $\widehat{\mathbf{b}}_{m}$

and the vertex and face numbering is made as previously. Let $\widehat{\mathbf{b}}_{m}$ be the center
of the edge $\widehat{\gamma}_{m}$, for $m=1, \ldots, 4$. The canonical basis $\left(\widehat{\mathbf{e}}_{1}, \widehat{\mathbf{e}}_{2}\right)$ of $\mathbb{R}^{2}$ can be simply
expressed with the vectors $\widehat{\mathbf{b}}_{m}$

$$
\widehat{\mathbf{e}}_{1}=-\widehat{\mathbf{b}}_{2}\left(=\widehat{\mathbf{b}}_{3}\right), \quad \widehat{\mathbf{e}}_{2}=-\widehat{\mathbf{b}}_{1} \quad\left(=\widehat{\mathbf{b}}_{4}\right)
$$

$$
\text { We choose }
$$

$$
\text { expressed with the vectors } \hat{b}_{m}
$$

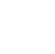

.
Since $K$ is assumed to be a nondegenerated quadrilateral, $\left(e_{1}, e_{2}\right)$ is a basis of $\mathbb{R}^{2}$

\author{
(1)
}

\section{Let $\widehat{K}=[-1,+1]^{2}$ be the reference square. The vertices of $\widehat{K}$ are denoted by $\widehat{\mathbf{a}}_{i}, 1 \leq i \leq 4$ and the faces are denoted by $\widehat{\gamma}_{m}, 1 \leq m \leq 4$.}


Let $B_{K}$ be the change of basis matrix given by

$$
B_{K} \widehat{\mathbf{e}}_{1}=\mathbf{e}_{1}, \quad B_{K} \widehat{\mathbf{e}}_{2}=\mathbf{e}_{2} .
$$

and $F_{K}^{\sharp}$ be the invertible affine mapping

$$
F_{K}^{\sharp}: \quad \widehat{\mathbf{x}} \in \mathbb{R}^{2} \rightarrow F_{K}^{\sharp}(\widehat{\mathbf{x}})=\mathbf{a}_{0}+B_{K} \widehat{\mathbf{x}} .
$$

This mapping $F_{K}^{\sharp}$ is the unique affine mapping such that

$$
F_{K}^{\sharp}\left(\widehat{\mathbf{b}}_{1}\right)=\mathbf{b}_{1}, \quad F_{K}^{\sharp}\left(\widehat{\mathbf{b}}_{2}\right)=\mathbf{b}_{2} .
$$

It is a bijection between $\widehat{K}$ and its image

$$
K^{\sharp}=F_{K}^{\sharp}(\widehat{K}) .
$$

As image of the reference square by an inversible affine mapping, $K^{\sharp}$ is a parallelogram. The associated parallelogram of $K$ being by definition the parallelogram which has the same face centers than $K$. We see that $K^{\sharp}$ is the associated parallelogram of $K$ and we have $K^{\sharp}=K$ if and only if $K$ is a parallelogram. Let

$$
K^{\vee}=\left(F_{K}^{\sharp}\right)^{-1}(K) .
$$

The parallelogram associated to the quadrilateral $K^{\vee}$ is the reference square $\widehat{K}$. To be able to make the analysis of quadrangular finite element ([1]) , we must precise the shape of the quadrangles that can be considered. In this purpose, we define in the next section the distortion of any element $K$ with respect to $K^{\sharp}$.

\subsection{Distortion parameters}

Let $\mathbf{d}$ be the vector of $\mathbb{R}^{2}$ given by

$$
\mathbf{d}=\frac{1}{4}\left(\mathbf{a}_{1}-\mathbf{a}_{2}-\mathbf{a}_{3}+\mathbf{a}_{4}\right) .
$$

We can interpret $2 \mathbf{d}$ as a vector whose endpoints are the midpoints of the diagonals of the quadrilateral $K$. This means that the quadrilateral $K$ is a parallelogram if and only if $\mathbf{d}=0$. It is easy to see that the vertices of $K^{\sharp}$ (the parallelogram associated to the quadrilateral $K$ ), are given by (see Figure 2)

$$
\mathbf{a}_{i}^{\sharp}=\mathbf{a}_{i}-s_{i} \mathbf{d}, \quad 1 \leq i \leq 4
$$

where

$$
s_{1}=s_{4}=+1, \quad s_{2}=s_{3}=-1 .
$$

We have $K=K^{\sharp}$ if and only if $\mathbf{d}=0$ in $\mathbb{R}^{2^{*}}$.

DeFinition 2.1 The vector $\mathbf{d}$ is named the distortion vector of $K$. 

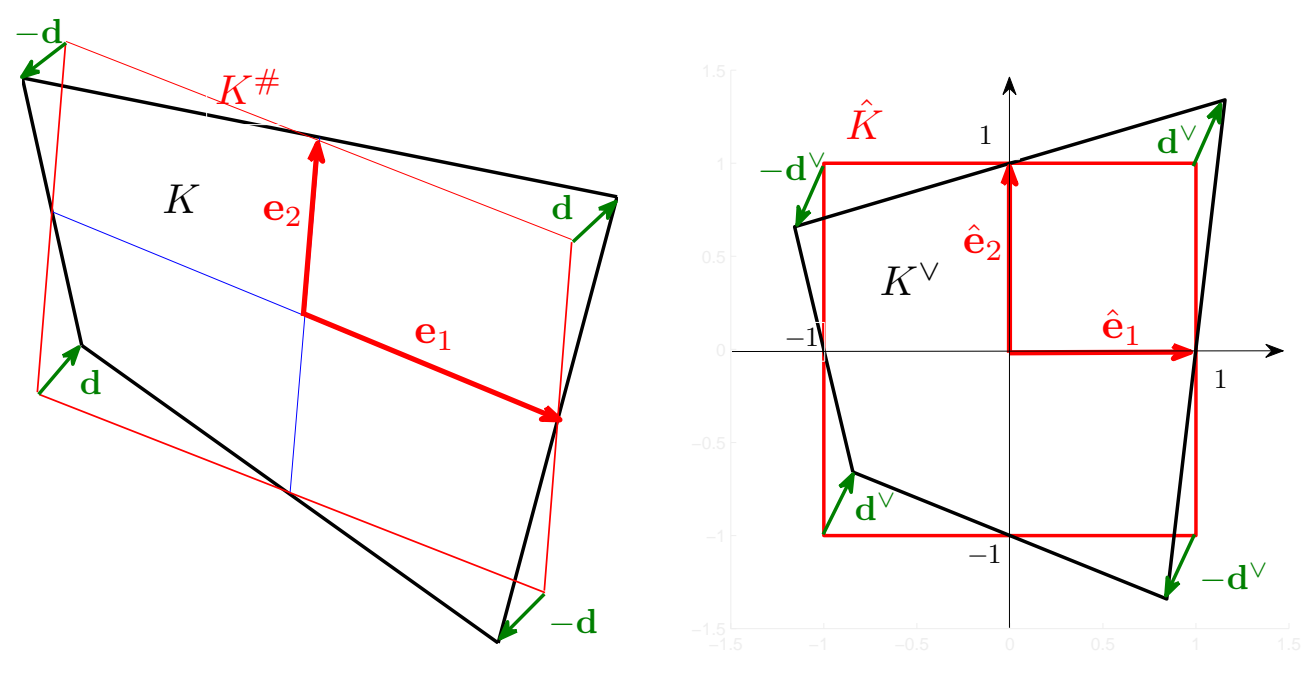

Figure 2. Distorsion vector

Let the distortion parameters, of $K, \delta_{1}$ and $\delta_{2}$ be such that

$$
\mathbf{d}=\delta_{1} \mathbf{e}_{1}+\delta_{2} \mathbf{e}_{2}
$$

where we recall that $\mathbf{e}_{m}$ is given by $\mathbf{e}_{m}=\mathbf{a}_{0}-\mathbf{b}_{m}, 1 \leqslant m \leqslant 2$. These parameters are invariant by affine mapping; in particular for the distortion vector $\mathbf{d}^{\vee}$ of $K^{\vee}$ we have

$$
\mathbf{d}^{\vee}=\delta_{1} \widehat{\mathbf{e}}_{1}+\delta_{2} \widehat{\mathbf{e}}_{2}
$$

Since the mapping $F_{K}^{\sharp}$ is invertible affine, $K$ is a convex quadrilateral if and only if $K^{\vee}$ is a convex quadrilateral. It is easy to show that $K$ is a convex quadrilateral if and only if we have

$$
\left|\delta_{1}\right|+\left|\delta_{2}\right|<1
$$

Then $K^{\vee}$ contains $B(\mathbf{0}, 1 / \sqrt{2})$ the ball centered at the origin and of radius $1 / \sqrt{2}$ and $K^{\vee}$ is contained in the square $[-2,+2]^{2}$. The quadrilateral $K$ is contained in the parallelogram

$$
K^{2 \sharp}=F_{K}^{\sharp}\left([-2,+2]^{2}\right) .
$$

This element $K^{2 \sharp}$ is homothetic to $K^{\sharp}$ with a ratio equal to 2 . Then, we have the inequality

$$
h_{K} \leq 2 h_{K^{\sharp}} .
$$

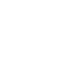


Last, we note that the Euclidean norm of the distortion vector of $K$ satisfies

$$
\frac{1}{4}\left(\sum_{1 \leq m \leq 2}\left|\delta_{m}\right|\right) \rho_{K^{\sharp}} \leq|\mathbf{d}| \leq \frac{1}{2}\left(\sum_{1 \leq m \leq 2}\left|\delta_{m}\right|\right) h_{K^{\sharp}} .
$$

Now, we give the definition of a family of regular meshes.

Definition 2.2 Regular Mesh

A family of quadrangular meshes is regular if and only if for each $K$ the distorsion parameters satisfy (4), and

$$
\exists \sigma>0 ; \quad \frac{h_{K^{\#}}}{\rho_{K^{\#}}} \leq \sigma .
$$

We can notice that this definition corresponds to the classical definition given by P.G. Ciarlet ([2]) when the $K$ 's are parallelogram.

\section{Local error estimates}

In this section we give some local error estimates without demonstration. The demonstration concerning the Lagrange finite elements can be found in ([10]) and with the same technics the results can be easily extended to the mixed finite elements.

\subsection{Interpolation error estimates}

\subsubsection{Lagrange interpolation error estimates}

Let $P_{K}$ be a finite dimensional vectorial space of polynomial functions defined over the quadrilateral $K$. We assume that the set $S_{K}=\left\{\mathbf{a}_{i}, 1 \leq i \leq 4\right\}$ is $P_{K^{-}}$ unisolvent. Then necessarly, $\operatorname{dim}\left(P_{K}\right)=4$. The basis functions of the Lagrange finite element $\left(K, P_{K}, S_{K}\right)$ are noted $p_{i, K}$ and the $P_{K}$-Lagrange interpolation operator is noted $\Pi_{K}$ : for every function $u$ defined on the vertices of $K$,

$$
\Pi_{K} u=\sum_{1 \leq i \leq 4} u\left(\mathbf{a}_{i}\right) p_{i, K}
$$

The basis functions $p_{i, K}$ are functions defined by definition on $K$; in fact, since they are polynomial, we consider them as functions defined on $K^{2 \sharp}$.

Proposition 3.1 Let us assume that the distortion parameters of $K$ satisfy (4), that the set $S_{K}$ is $P_{K}$-unisolvent and that the inclusion $P_{1}(K) \subset P_{K}$ holds. Moreover, let $r$ be an integer sufficiently large for the the inclusion $P_{K} \subseteq P_{r}(K)$ to hold. Then there exists a constant $c_{r}$, which depends only on $r$, such that for every $u \in H^{2}(K)$,

$$
\left\|u-\Pi_{K} u\right\|_{1, K} \leq c_{r} \frac{h_{K^{\sharp}}^{2}}{\rho_{K^{\sharp}}}\left(\sum_{1 \leq i \leq 4}\left\|p_{i, K}\right\|_{0, \infty, K^{2 \sharp}}\right)|u|_{2, K} .
$$




\subsubsection{Hdiv interpolation error estimates}

Let $\Psi_{K}=P_{K} \times P_{K}$ be a finite dimensional vectorial space of polynomial functions defined over the quadrilateral $K$. We assume that the set $\Sigma_{K}=$ $\left\{\mathbf{w} \rightarrow \frac{1}{\left|\gamma_{m}\right|} \int_{\gamma_{m}} \mathbf{w} \cdot \mathbf{n} d \sigma ; 1 \leq m \leq 4\right\}$ is $\Psi_{K}$-unisolvent. The basis functions of the mixed finite element $\left(K, \Psi_{K}, \Sigma_{K}\right)$ are noted $\psi_{m, K}$ and the $\Psi_{K}-$ Withney interpolation operator is noted $\Pi_{K}^{W}$ : for every function $\mathbf{p}$ such that $\int_{\gamma_{m}} \mathbf{p . n} d \sigma ; 1 \leq m \leq 4$ exist

$$
\Pi_{K}^{W} \mathbf{p}=\sum_{1 \leq m \leq 4} \frac{1}{\left|\gamma_{m}\right|} \int_{\gamma_{m}} \mathbf{p . n} d \sigma \psi_{m, K}
$$

The basis functions $\psi_{m, K}$ are considered as functions defined on $K^{2 \sharp}$.

Proposition 3.2 Let us assume that the distortion parameters of $K$ satisfy (4), that the set $\Sigma_{K}$ is $P_{K} \times P_{K}$-unisolvent and that the inclusion $P_{0}(K)^{2} \oplus \mathbf{x} P_{0}(K) \subset$ $P_{K} \times P_{K}$ holds. Moreover, let $r$ be an integer sufficiently large for the the inclusion $P_{K} \times P_{K} \subseteq P_{r}(K) \times P_{r}(K)$ to hold. Then there exists a constant $c_{r}$, which depends only on $r$, such that for every $\mathbf{p} \in H^{1}(K)$,

$$
\left\|\mathbf{p}-\Pi_{K}^{W} \mathbf{p}\right\|_{H(d i v, K)} \leq c_{r} \frac{h_{K^{\sharp}}^{2}}{\rho_{K^{\sharp}}}\left(\sum_{1 \leq m \leq 4}\left\|\psi_{m, K}\right\|_{0, \infty, K^{2 \sharp}}\right)|\mathbf{p}|_{1, K} \cdot
$$

Proposition 3.3 We suppose that there exists an integer $r$ sufficiently large such that $P_{K} \times P_{K} \subseteq P_{r}(K) \times P_{r}(K)$. Then, for every $\mathbf{q}_{h} \in P_{K} \times P_{K}$ there exists a constant $c_{r}$ depending only on $r$ such that

$$
\left\|\mathbf{q}_{h}\right\|_{1, K^{2 \sharp}} \leq c_{r} \frac{1}{\rho_{K^{\sharp}}}\left\|\mathbf{q}_{h}\right\|_{0, K^{2 \sharp}} .
$$

\section{$3.2 \quad$ error face estimates}

For $u \in H^{1}(K)$ we note $\pi_{\gamma_{m}}^{k} u$ the best approximation of the trace of $u$ in $L^{2}\left(\gamma_{m}\right)$ by a polynomial $P_{k}$.

Lemma 3.4 Assume (4); then there exists a constant $C$, independent of the distortion parameters, such that for every $\left.u \in H^{1}\left(K^{\vee}\right)\right)$ and every $m$ with $1 \leq m \leq 4$

$$
\|u\|_{0, \gamma_{m}^{\vee}} \leq C\|u\|_{1, K^{\vee}}
$$

Proposition 3.5 Assume that the distortion parameters of $K$ satisfy (4). Then there exists a constant $C$, independent of the geometry of $K$, such that: $\forall u \in$ $H^{1}(K)$ and $\forall m$ with $1 \leq m \leq 4$, we have

$$
\left\|u-\pi_{\gamma_{m}}^{0} u\right\|_{0, \gamma_{m}} \leq C h_{K^{\sharp}}^{1 / 2}\left(\frac{h_{K^{\sharp}}}{\rho_{K^{\sharp}}}\right)^{1 / 2}|u|_{1, K} \cdot
$$
$K$.

We can notice that $\pi_{\gamma_{m}}^{0} u$ corresponds to the mean value of $u$ on the face $\gamma_{m}$ of Proposition 3.6 Assume that the distortion parameters of $K$ satisfy (4). Then there exists a constant $C$, independent of the geometry of $K$, such that: $\forall u \in$ 
$H^{2}(K)$ and $\forall m$ with $1 \leq m \leq 4$, we have

$$
\left\|u-\pi_{\gamma_{m}}^{1} u\right\|_{0, \gamma_{m}} \leq C h_{K^{\sharp}}^{3 / 2}\left(\frac{h_{K^{\sharp}}}{\rho_{K^{\sharp}}}\right)^{1 / 2}|u|_{2, K} \cdot
$$

\section{The model problem and the patch tests}

We consider the second order elliptic model problem:

$$
\left\{\begin{aligned}
-\operatorname{div}(A \operatorname{grad} u) & =f & & \text { in } \Omega \\
u & =0 & & \text { on } \Gamma .
\end{aligned}\right.
$$

where $A=\left(a_{i, j}\right)$ is a symmetric matrix satisfying

$$
\forall x \in \bar{\Omega}, \forall \xi \in \mathbb{R}^{2}, \quad c \sum_{i=1}^{2} \xi_{i}^{2} \leq \sum_{i, j=1}^{2} a_{i, j}(x) \xi_{i} \xi_{j} \leq c^{-1} \sum_{i=1}^{2} \xi_{i}^{2},
$$

and $\Gamma:=\partial \Omega$ is the boundary of a polyhedral domain $\Omega \subset \mathbb{R}^{2}$. Let $\mathcal{T}_{h}$ be a triangulation of $\Omega$ into quadrilaterals. Let $\partial \mathcal{T}_{h}$ denotes the set of the edgess of the elements of $\mathcal{T}_{h}$ and $\partial \mathcal{T}_{h} \backslash \partial \Omega$ denotes the set of interior edges. For each element $\gamma$ of $\partial \mathcal{T}_{h} \backslash \partial \Omega$, there exist $K^{+}$and $K^{-}$in $\mathcal{T}_{h}$ such that $\bar{K}^{+} \cap \bar{K}^{-}=\gamma$. The unitary outward normal of $K^{+}$is noted $\mathbf{n}^{+}$and the normal of a face is defined by $\mathbf{n}=\mathbf{n}^{+}$. For each subset $\gamma$ of $\partial \Omega, \mathbf{n}$ denotes the unitary outward normal of $\Omega$.

\subsection{Variational formulation and error estimate}

The variational problem associated to (10) is: find $u \in H_{0}^{1}(\Omega)$ such that

$$
\forall v \in H_{0}^{1}(\Omega) \quad \int_{\Omega} A \operatorname{grad} u \cdot \operatorname{grad} v d x=\int_{\Omega} f v d x .
$$

We consider

$$
V_{\mathcal{T}_{h}}=\left\{v \in L^{2}(\Omega) ;\left.v\right|_{K} \in H^{1}(K) \text { for each } K \in \mathcal{T}_{h}\right\}
$$

and

$$
V_{h}=\left\{v_{h} \in L^{2}(\Omega) ;\left.v_{h}\right|_{K} \in P_{K} \text { for each } K \in \mathcal{T}_{h}\right\} .
$$

Where $P_{K}$ is a polynomial space. A non conforming finite element method for problem (11) is: find $u_{h} \in V_{h}$ such that

$$
\forall v_{h} \in V_{h}, \quad \sum_{K \in \mathcal{T}_{h}} \int_{K} A \operatorname{grad} u_{h} \cdot \operatorname{grad} v_{h} d x=\int_{\Omega} f v_{h} d x .
$$

For $v, w \in H_{0}^{1}(\Omega)+V_{\mathcal{T}_{h}}$ we define

$$
a_{h}(v, w)=\sum_{K \in \mathcal{T}_{h}} \int_{K} A \operatorname{grad} \cdot v \operatorname{grad} w d x
$$


and

$$
\|v\|_{1, h}=\left(\sum_{K \in \mathcal{T}_{h}}\|v\|_{1, K}^{2}\right)^{1 / 2},|v|_{1, h}=\left(\sum_{K \in \mathcal{T}_{h}}|v|_{1, K}^{2}\right)^{1 / 2}
$$

Let us define the jump of $w_{h} \in V_{h}$ on $\gamma$. If $\gamma$ is in $\partial \mathcal{T}_{h} \backslash \partial \Omega$ then $\left[w_{h}\right]=w_{h}^{+}-w_{h}^{-}$ where $w_{h}^{ \pm}$is the trace on $\gamma$ of $w_{h}^{ \pm} \in H^{1}\left(K^{ \pm}\right)$else $\left[w_{h}\right]$ represents the trace on $\gamma$ of $w_{h} \in H^{1}(K)$.

Since $a_{h}(.,$.$) is uniformly V_{\mathcal{T}_{h}}$ elliptic, the following basic error estimate (approximation and consistency error) hold (see [1], [6], [8] ).

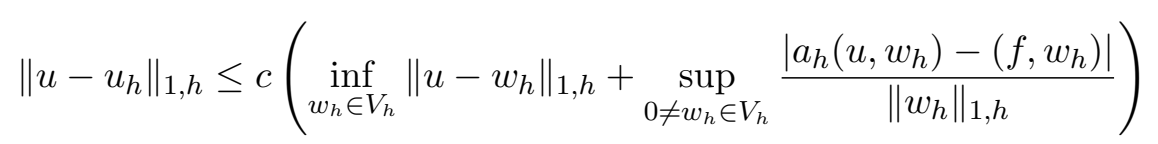

with

$$
a_{h}\left(u, w_{h}\right)-\left(f, w_{h}\right)=\sum_{\gamma \in \mathcal{T}_{h}} \int_{\gamma} \frac{\partial u}{\partial n}\left[w_{h}\right] d s
$$

We suppose that the solution $u$ of $(10)$ is in $H^{2}(\Omega)$ and the mesh is regular.

Proposition 4.1 Approximation error

First of all we assume that for any $K$, the set $\left\{a_{i}, 1 \leq i \leq 4\right\}$ is $P_{K}$-unisolvent. Secondly we suppose that $\exists r>0 \forall K P_{1}(K) \subset P_{K} \subset P_{r}(K)$. Moreover we assume that the basis functions $p_{i, K}$ of $P_{K}$ satisfy $\exists C>0\left\|p_{i, K}\right\|_{0, \infty, K^{2 \#}}<C$.

Under these three assumptions, we have

$$
\inf _{w_{h} \in V_{h}}\left\|u-w_{h}\right\|_{1, h} \leq C h|u|_{2, \Omega} .
$$

Proof Using Proposition 3.1 , we have immediatly

$$
\left(\sum_{K \in \mathcal{T}_{h}}\left\|u-\Pi_{K} u\right\|_{1, h}\right)^{1 / 2} \leq C h|u|_{2, \Omega}
$$

where $C$ is a constant independent of the mesh.

Proposition 4.2 Consistency error

We suppose that the Patch Test is satisfied, namely

$$
\forall w_{h} \in V_{h}, \quad \forall \gamma \in \partial \mathcal{T}_{h} \quad \int_{\gamma}\left[w_{h}\right] d s=0
$$

then

$$
\left|a_{h}\left(u, w_{h}\right)-\left(f, w_{h}\right)\right| \leq C h|u|_{2, \Omega}\left\|w_{h}\right\|_{1, h}
$$

Proof Let $\gamma$ be in $\partial \mathcal{T}_{h} \backslash \partial \Omega$, we have $\int_{\gamma} \frac{\partial u}{\partial \mathbf{n}}\left[w_{h}\right] d \sigma=\int_{\gamma} \frac{\partial u}{\partial \mathbf{n}^{+}}\left(w_{h}^{+}-w_{h}^{-}\right) d \sigma$ where $w_{h}$ satisfies $\int_{\gamma} w_{h^{+}} d \sigma=\int_{\gamma} w_{h^{-}} d \sigma$. Therefore, dividing by $|\gamma|$, we have ${\overline{w_{h}^{+}}}^{\gamma}=\overline{w_{h}^{-}} \gamma$. 
Thus, we obtain

$$
\int_{\gamma} \frac{\partial u}{\partial \mathbf{n}}\left[w_{h}\right] d \sigma=\int_{\gamma} \frac{\partial u}{\partial \mathbf{n}^{+}}\left(w_{h}^{+}-{\overline{w_{h}^{+}}}^{\gamma}\right) d \sigma-\int_{\gamma} \frac{\partial u}{\partial \mathbf{n}^{-}}\left(w_{h}^{-}-{\overline{w_{h}^{-}}}^{\gamma}\right) d \sigma .
$$

Obviously, for each constant $c$ we have

and finally

$$
\left|\int_{\gamma} \frac{\partial u}{\partial \mathbf{n}}\left[w_{h}\right] d \sigma\right| \leq C h\left(|u|_{2, K^{+}}\left|w_{h}\right|_{1, K^{+}}+|u|_{2, K^{-}}\left|w_{h}\right|_{1, K^{-}}\right) .
$$

The result, for each $\gamma \subset \partial \Omega$, is similar

$$
\left|\int_{\gamma} \frac{\partial u}{\partial \mathbf{n}}\left[w_{h}\right] d \sigma\right| \leq C h|u|_{2, K}\left|w_{h}\right|_{1, K} .
$$

Summing on all faces $\gamma_{m}$, the right hand side of the inequality on each element $K$ appears at most 4 times. Thus, the expected results hold.

Consequently, the pseudo-conforming Lagrange finite element method converges
with the order 1 .

Consequently, the pseudo-conforming Lagrange finite element method converges
with the order 1 .

\subsection{Mixed formulation and error estimate}

In the classical mixed formulation ([3], [5]) we introduce the new variable $\mathbf{p}=$ $A \operatorname{grad} u$ and the mixed variational formulation for (10) is: find $u \in L^{2}(\Omega)$ and $\mathbf{p} \in H(\operatorname{div}, \Omega)$ such that

$$
\begin{array}{cl}
\int_{\Omega} \operatorname{div} \mathbf{p} v d x-\int_{\Omega} f v d x=0 & \forall v \in L^{2}(\Omega), \\
\int_{\Omega} A^{-1} \mathbf{p . q}-\int_{\Omega} u \operatorname{div} \mathbf{q} d x=0 & \forall \mathbf{q} \in H(\operatorname{div}, \Omega) .
\end{array}
$$

We consider

$$
\begin{gathered}
M_{h}=\left\{v_{h} \in L^{2}(\Omega) ;\left.v_{h}\right|_{K} \in P_{0} \text { for each } K \in \mathcal{T}_{h}\right\} \\
L_{\mathcal{T}_{h}}=\left\{\mathbf{q} \in L^{2}(\Omega) \times L^{2}(\Omega) ;\left.\mathbf{q}\right|_{K} \in H(\text { div }, K) \text { for each } K \in \mathcal{T}_{h}\right\}
\end{gathered}
$$

We consider$$
\text { (2) }
$$

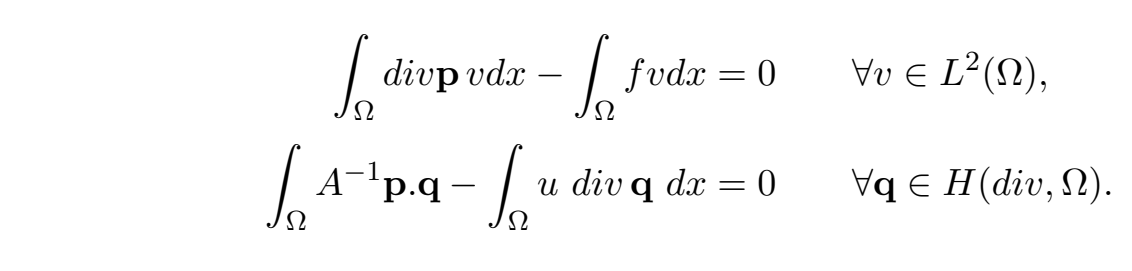
(1) (1)

. 
and

$$
L_{h}=\left\{\mathbf{q}_{h} \in L^{2}(\Omega) \times L^{2}(\Omega) ;\left.\mathbf{q}_{h}\right|_{K} \in P_{K} \times P_{K} \text { for each } K \in \mathcal{T}_{h}\right\},
$$

where $P_{K}$ is a polynomial space.

For $\mathbf{p} \in H(\operatorname{div}, \Omega)+\mathcal{T}_{h}$, we define

$$
\|\mathbf{p}\|_{H \operatorname{div}(0, h)}^{2}=\sum_{K \in \mathcal{T}_{h}}\|\mathbf{p}\|_{H(\operatorname{div}, K)}^{2} .
$$

Let us notice that the space $M_{h}$ is defined as usually, and $L_{\mathcal{T}_{h}} \nsubseteq H(\operatorname{div}, \Omega)$. Therefore the non-conformity to study concerns the approximation of $\mathbf{q}$.

For any $(u, \mathbf{p}, \mathbf{q}) \in L^{2}(\Omega) \times H(\operatorname{div}, \Omega) \times H(\operatorname{div}, \Omega)+L^{2}(\Omega) \times L_{\mathcal{T}_{h}} \times L_{\mathcal{T}_{h}}$ we define

$$
c_{h}(u, \mathbf{p}, \mathbf{q})=\sum_{K \in \mathcal{T}_{h}} \int_{K} A^{-1} \mathbf{p . q}-\sum_{K \in \mathcal{T}_{h}} \int_{K} u \operatorname{div} \mathbf{q} d x
$$

A non conforming mixed finite element method for problem (11) is: find $u_{h} \in M_{h}$ and $\mathbf{q}_{h} \in L_{h}$ such that

$$
\begin{aligned}
\sum_{K \in \tau_{h}} \int_{K} v_{h} \operatorname{div} \mathbf{p}_{h} d x-\sum_{K \in \tau_{h}} \int_{K} f v_{h} d x=0 & \forall v_{h} \in M_{h}, \\
\sum_{K \in \tau_{h}} \int_{K} A^{-1} \mathbf{p}_{h} \cdot \mathbf{q}_{h}-\sum_{K \in \tau_{h}} \int_{K} u_{h} \operatorname{div} \mathbf{q}_{h} d x=0 & \forall \mathbf{q}_{h} \in L_{h} .
\end{aligned}
$$

If the following inf - sup condition is satisfyied:

$$
\inf _{\left\{v_{h} \in M_{h} ;\left\|u_{h}\right\|_{0, \Omega}=1\right\}} \sup _{\left\{\mathbf{q}_{h} \in L_{h},\left\|\mathbf{q}_{h}\right\|_{H d i v(0, h)}=1\right\}} b\left(u_{h}, \mathbf{q}_{h}\right) \geq \beta>0
$$

where $b\left(u_{h}, \mathbf{q}_{h}\right)=\sum_{K \in \tau_{h}} \int_{K} u_{h} d i v \mathbf{q}_{h} d x$, then the problem (16),(17) admits a unique solution and we have the a priori error estimate

$$
\begin{aligned}
& \left\|\mathbf{p}-\mathbf{p}_{h}\right\|_{H \operatorname{div}(0, h)}+\left\|u-u_{h}\right\|_{0, h} \leq \\
& c\left(\inf _{v_{h} \in M_{h}}\left\|u-v_{h}\right\|_{0, h}+\inf _{\mathbf{q}_{h} \in L_{h}}\left\|\mathbf{p}-\mathbf{q}_{h}\right\|_{H \operatorname{div}(0, h)}+\sup _{\substack{0 \neq \mathbf{q}_{h} \in L_{h}\\
}} \frac{\left|c_{h}\left(u, \mathbf{p}, \mathbf{q}_{h}\right)\right|}{\left\|\mathbf{q}_{h}\right\|_{0, h}}\right) .
\end{aligned}
$$

Using the Green formula : $\int_{K} u d i v\left(\mathbf{q}_{h}\right) d x=-\int_{K} \operatorname{grad} u \cdot \mathbf{q}_{h} d x+\int_{\partial K} u \mathbf{q}_{h} \cdot \mathbf{n} d \sigma$ we prove that

$$
c_{h}\left(u, \mathbf{p}, \mathbf{q}_{h}\right)=\sum_{\gamma \in \partial \mathcal{T}_{h}} \int_{\gamma} u\left[\mathbf{q}_{h} \cdot \mathbf{n}\right] d \sigma .
$$

Now we suppose that the solution $u$ of $(10)$ is in $H^{2}(\Omega)$ and the mesh is regular.

Proposition 4.3 inf - sup condition

We assume first that for all $K, \Sigma_{K}$ is $\Psi_{K}$-unisolvent. Secondly we suppose that for each $\mathbf{q}_{h} \in L_{h}$, div $\left(\mathbf{q}_{h}\right) \in M_{h}$. Moreover we assume that the basis functions $\psi_{m, K}$ 
of $\Psi_{K}$ satisfy $\exists C>0\left\|\psi_{m, K}\right\|_{0, \infty, K^{2 \#}}<C$.

Under these three assumptions the inf - sup condition (18) holds.

Proof Since the domain $\Omega$ is regular, the inf - sup condition on the continuous problem (cf [3], [5]) gives :

For each $u \in L^{2}(\Omega)$, there exists $\mathbf{p} \in H^{1}(\Omega)$ such that $\operatorname{div}(\mathbf{p})=u$ and the estimate $\|\mathbf{p}\|_{1, \Omega} \leq C\|u\|_{0, \Omega}$ holds with a constant $C$ independant of the mesh.

Therefore, this property is true for each $u_{h} \in M_{h}$. Let $\mathbf{p}_{h}=\Pi_{h}^{W} \mathbf{p}$ be the Withneyinterpolant of $\mathbf{p}$ in $L_{h}$ and we want to prove that $\left\|\mathbf{p}_{h}\right\|_{H \operatorname{div}(0, h)} \leq C\left\|u_{h}\right\|_{0, \Omega}$. Using the assumption 3 , we have $\operatorname{div}\left(\mathbf{p}_{h}\right)=\operatorname{div}(\mathbf{p})$ on each $K$. Furthermore, for each $\mathbf{x}$ in $K$, we have

$$
\left|\mathbf{p}_{h}(\mathbf{x})\right| \leq \sum_{1 \leq m \leq 4} \frac{1}{\left|\gamma_{m}\right|}\left|\int_{\gamma_{m}} \mathbf{p . n} d \sigma\right|\left|\psi_{m, K}(\mathbf{x})\right|
$$

then

$$
\left\|\mathbf{p}_{h}\right\|_{0, K} \leq \sum_{1 \leq m \leq 4}\|\mathbf{p} . \mathbf{n}\|_{0, \gamma_{m}} \sum_{1 \leq m \leq 4}\left\|\psi_{m, K}\right\|_{0, \infty, K}
$$

and using lemma 3.4 and the assumption 2 we obtain

$$
\begin{aligned}
& \left\|\mathbf{p}_{h}\right\|_{0, K} \leq C\|\mathbf{p}\|_{1, K} \\
& \text { have } \\
& \sum_{1 \leq m \leq 4} \frac{1}{\left|\gamma_{m}\right|}\left|\int_{\gamma_{m}} \mathbf{p . n} d \sigma\right|\left|\operatorname{div}\left(\psi_{m, K}(\mathbf{x})\right)\right|
\end{aligned}
$$

Equally, for each $\mathbf{x}$ in $K$, we have

$$
\begin{aligned}
& \qquad\left\|\mathbf{p}_{h}\right\|_{0, K} \leq C\|\mathbf{p}\|_{1, K} \\
& \text { each } \mathbf{x} \text { in } K, \text { we have } \\
& \left|\operatorname{div}\left(\mathbf{p}_{h}(\mathbf{x})\right)\right| \leq \sum_{1 \leq m \leq 4} \frac{1}{\left|\gamma_{m}\right|}\left|\int_{\gamma_{m}} \mathbf{p . n} d \sigma\right|\left|\operatorname{div}\left(\psi_{m, K}(\mathbf{x})\right)\right|
\end{aligned}
$$

$$
\left\|\operatorname{div} \mathbf{p}_{h}\right\|_{0, K} \leq C\|\mathbf{p}\|_{1, K}
$$

Finally we have

(1)

(1)

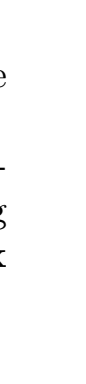

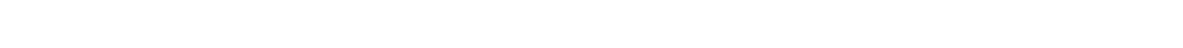

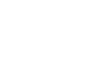

$\left|\operatorname{div}\left(\mathbf{p}_{h}(\mathbf{x})\right)\right| \leq \sum_{1 \leq m \leq 4} \frac{1}{\left|\gamma_{m}\right|}\left|\int_{\gamma_{m}} \mathbf{p . n} d \sigma\right|\left|\operatorname{div}\left(\psi_{m, K}(\mathbf{x})\right)\right|$
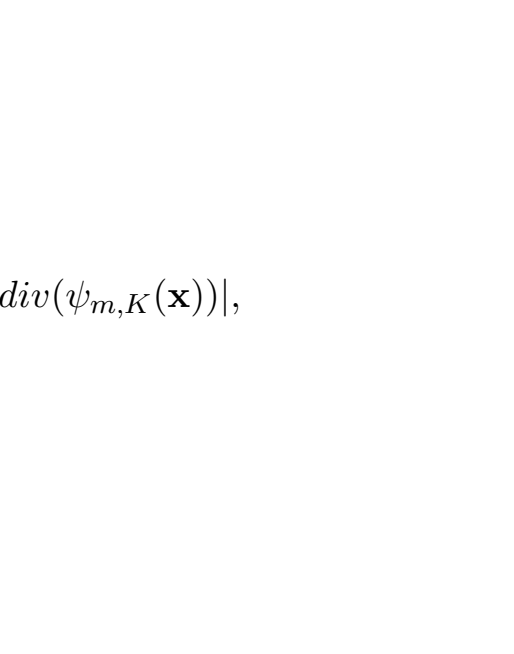
since $\operatorname{div}\left(\psi_{m, K}(\mathbf{x})\right)$ is constant on $K$, we deduce
since div $\left(\varphi_{m}, K(\mathrm{x})\right)$ is

(1) 
Under these three assumptions we have

$$
\inf _{\mathbf{q}_{h} \in L_{h}}\left\|\mathbf{p}-\mathbf{q}_{h}\right\|_{H \operatorname{div}(0, h)} \leq C h|u|_{2, \Omega} .
$$

Proof Since $\mathbf{p}=A \operatorname{grad} u$ and using Proposition 3.2, we have immediatly

$$
\left(\sum_{K \in \mathcal{T}_{h}}\left\|\mathbf{p}-\Pi_{K}^{W} \mathbf{p}\right\|_{H(d i v, K)}\right)^{1 / 2} \leq C h|A \operatorname{grad} u|_{1, \Omega} \leq C h|u|_{2, \Omega}
$$

where $C$ is a constant independent of the mesh.

Remark: The approximation error on $u_{h}$ does not raise problem and is bounded by $C h|u|_{1, \Omega}$.

Proposition 4.5 Consistency error

If the Patch Test conditions are satisfied, namely

$$
\forall \mathbf{q}_{h} \in V_{h}, \quad \forall \gamma \in \partial \mathcal{T}_{h} \quad \int_{\gamma}\left[\mathbf{q}_{h} \cdot \mathbf{n}\right] d \sigma=0
$$

and

$$
\forall \mathbf{q}_{h} \in V_{h}, \quad \forall \gamma \in \partial \mathcal{T}_{h} \quad \int_{\gamma} \sigma\left[\mathbf{q}_{h} \cdot \mathbf{n}\right] d \sigma=0
$$

then

$$
\left|c_{h}\left(u, \mathbf{p}, \mathbf{q}_{h}\right)\right| \leq C h|u|_{2, \Omega}\left\|w_{h}\right\|_{1, h} .
$$

Proof Let $\gamma$ be in $\partial \mathcal{T}_{h} \backslash \partial \Omega$, we have $\int_{\gamma} u\left[\mathbf{q}_{h} \cdot \mathbf{n}\right] d \sigma=\int_{\gamma} u\left(\mathbf{q}_{h}^{+} \cdot \mathbf{n}^{+}-\mathbf{q}_{h}^{-} \cdot \mathbf{n}^{-}\right) d \sigma$.

Since $\int_{\gamma} \mathbf{q}_{h}^{+} \cdot \mathbf{n}^{+} d \sigma=\int_{\gamma} \mathbf{q}_{h}^{-} \cdot \mathbf{n}^{-} d \sigma$ we have $\pi_{\gamma}^{0}\left(\mathbf{q}_{h}^{+} \cdot \mathbf{n}^{+}\right)=\pi_{\gamma}^{0}\left(\mathbf{q}_{h}^{-} \cdot \mathbf{n}^{-}\right)$.

Consequently,

$\int_{\gamma} u\left(\mathbf{q}_{h}^{+} \cdot \mathbf{n}^{+}-\mathbf{q}_{h}^{-} \cdot \mathbf{n}^{-}\right) d \sigma=\int_{\gamma} u-\left(\mathbf{q}_{h}^{+} \cdot \mathbf{n}^{+}-\pi_{\gamma}^{0}\left(\mathbf{q}_{h}^{+} \cdot \mathbf{n}^{+}\right)\right) d \sigma-\int_{\gamma} u-\left(\mathbf{q}_{h}^{-} \cdot \mathbf{n}^{-}-\pi_{\gamma}^{0}\left(\mathbf{q}_{h}^{-} \cdot \mathbf{n}^{-}\right)\right) d \sigma$.

So, for each constant $c_{0}$ and $c_{1}$ we have

$$
\int_{\gamma} u\left(\mathbf{q}_{h}^{+} \cdot \mathbf{n}^{+}-\pi_{\gamma}^{0}\left(\mathbf{q}_{h}^{+} \cdot \mathbf{n}^{+}\right)\right) d \sigma=\int_{\gamma}\left(u-c_{0}+c_{1} \sigma\right)\left(\mathbf{q}_{h}^{+} \cdot \mathbf{n}^{+}-\pi_{\gamma}^{0}\left(\mathbf{q}_{h}^{+} \cdot \mathbf{n}^{+}\right)\right) d \sigma
$$

and

$$
\left|\int_{\gamma}\left(u-\pi_{\gamma}^{1} u\right)\left(\mathbf{q}_{h}^{+} \cdot \mathbf{n}^{+}-\pi_{\gamma}^{0}\left(\mathbf{q}_{h}^{+} \cdot \mathbf{n}^{+}\right)\right) d \sigma\right| \leq\left\|u-\pi_{\gamma}^{1} u\right\|_{0, \gamma}\left\|\mathbf{q}^{+}-\pi_{\gamma}^{0}\left(\mathbf{q}_{h}^{+} \cdot \mathbf{n}^{+}\right)\right\|_{0, \gamma} .
$$

From Propositions 3.5, 3.6 and 3.3 we deduce that

$$
\begin{aligned}
\left|\int_{\gamma} u \mathbf{q}_{h}^{+} \cdot \mathbf{n}^{+} d \sigma\right| & \leq c h^{2}\left\|\mathbf{q}_{h}^{+}\right\|_{1, K^{+}}|u|_{2, K^{+}} \\
& \leq c h\left\|\mathbf{q}_{h}^{+}\right\|_{0, K^{+2 \sharp}}|u|_{2, K+}
\end{aligned}
$$


and finally

$$
\left|\int_{\gamma} u\left[\mathbf{q}_{h} \cdot \mathbf{n}\right] d \sigma\right| \leq \operatorname{ch}\left(\left\|\mathbf{q}_{h}^{+}\right\|_{0, K^{+2 \sharp}}|u|_{2, K^{+}}+\left\|\mathbf{q}_{h}^{-}\right\|_{0, K^{-2 \sharp}}|u|_{2, K^{-}}\right) .
$$

We sum on all the faces $\gamma$. In the right hand side of the inequality an element $K$ appears at most 4 times, so we have

$$
\begin{aligned}
\left|\sum_{\gamma \in \partial \mathcal{T}_{h}} \int_{\gamma} u\left[\mathbf{q}_{h} \cdot \mathbf{n}\right] d \sigma\right| & \leq \operatorname{ch}\left(\sum_{K \in \tau_{h}}\left\|\mathbf{q}_{h}\right\|_{0, K^{2 \sharp}}\right)|u|_{2, \Omega} \\
& \leq \operatorname{ch}\left\|\mathbf{q}_{h}\right\|_{0, \Omega}|u|_{2, \Omega} .
\end{aligned}
$$

Consequently, the pseudo-conforming mixed finite element converges with order 1 .

\section{Polynomial finite elements}

This section is devoted to the construction of polynomial finite elements on quadrilaterals satisfying the assumtions used in 4 . In the first subsection we study the case of Lagrange finite elements and in the second the case of Raviart-Thomas finite elements. For each finite element we give explicitly the basic functions.

\subsection{Lagrange finite elements}

Note that $P_{k}=\left\{q=q^{\vee} \circ\left(F_{K}^{\sharp}\right)^{-1} ; q^{\vee} \in P_{k}\right\}$. The same property is not true for the space $Q_{k}$. Therefore we introduce the space:

$$
Q_{k}^{K}=\left\{q^{\vee} \circ\left(F_{K}^{\sharp}\right)^{-1} ; q^{\vee} \in Q_{k}\right\}
$$

which is a subspace of $P_{2 k}$.

If we choose $P_{K}=Q_{1}^{K}$ then $\left(K, P_{K}, S_{K}\right)$ is a finite element, but the approximation $u_{h}$ of the solution of (10) obtained with this element does not converge without additional assumptions (see the numerical results in the next section). Indeed the basic functions of the space $V_{\mathcal{T}_{h}}$ are discontinuous on the faces of the elements, and we loose the order of convergence on the consistency error term.

Therefore our goal is to build polynomial finite elements on quadrilaterals satisfying the assumptions of Propositions 4.1 and 4.2.

Since the trapezoidal formula is exact for each polynomial of order 1, we have

$$
\forall q \in P_{1}, \int_{\gamma_{m}} q d \sigma=\frac{1}{2}\left|\gamma_{m}\right| \sum_{a_{i} \in \gamma_{m}} q\left(a_{i}\right), \text { for all } m=1, \ldots, 4
$$

where $\left|\gamma_{m}\right|$ is the length of the edge $\gamma_{m}$. 
Let $P_{K}$ be the following polynomial space:

$$
P_{K}=\left\{q \in Q_{2}^{K} \cap P_{3} ; \int_{\gamma_{m}} q d \sigma=\frac{1}{2}\left|\gamma_{m}\right| \sum_{a_{i} \in \gamma_{m}} q\left(a_{i}\right) \text {, for all } m=1, \ldots, 4\right\}
$$

The Simpson formula integrates exactly the cubic functions on each edge and consequently the space $P_{K}$ can be defined as well as

$$
P_{K}=\left\{q \in Q_{2}^{K} \cap P_{3} ; q\left(b_{m}\right)=\frac{1}{2} \sum_{a_{i} \in \gamma_{m}} q\left(a_{i}\right), \text { for all } m=1, \ldots, 4\right\} .
$$

Proposition 5.1 For any convex quadrilateral $K$, the triad $\left(K, P_{K}, S_{K}\right)$ is a Lagrange finite element.

Proof Let us introduce $a_{i}^{\vee}=b_{i-4}^{\vee}$ and $\widehat{a}_{i}=\widehat{b}_{i-4}$ for $i=5, \ldots, 8$. Using the invertible affine mapping $F_{K}^{\sharp}$, we only need to prove that:

For each distortion parameters $\delta=\left(\delta_{1}, \delta_{2}\right)$ such that $\left|\delta_{1}\right|+\left|\delta_{2}\right|<1$ the unique function $q \in Q_{2}^{K} \cap P_{3}$ satisfying $\left(q^{\vee}\left(a_{i}^{\vee}\right)=0 ; 1 \leq i \leq 8\right)$ is $q \equiv 0$.

Let us introduce the polynomials $r_{j} \in Q_{2} \cap P_{3}$ satisfying

$$
r_{j}\left(\widehat{a}_{i}\right)=\delta_{i, j}
$$

and the square matrix $R$ of order 8 defined by $R_{i, j}=r_{j}\left(a_{i}^{\vee}\right)$. Both symbolic calculus and an explicit calculus give

$$
\operatorname{det} R=\left(1-\delta_{1}^{2}\right)\left(1-\delta_{2}^{2}\right)\left(1-\left(\delta_{1}+\delta_{2}\right)^{2}\right)\left(1-\left(\delta_{1}-\delta_{2}\right)^{2}\right)
$$

Since $\left|\delta_{1}\right|+\left|\delta_{2}\right|<1$ then $\operatorname{det} R>0$. Therefore $R$ is invertible and $q \equiv 0$. Note that if $\delta=0$ the $r_{j}$ 's correspond to the basis of the serendipity finite element and in this case $P_{K} \equiv Q_{1}^{K}$.

Using the matrix $R$, we can calculate explicitly the image by $F_{K}^{\sharp}$ of the 4 basis functions of $P_{K}$

$$
\begin{aligned}
& p_{1, K}^{\vee}\left(x_{1}^{\vee}, x_{2}^{\vee}\right)=\frac{1}{4}\left(1+x_{1}^{\vee}-x_{2}^{\vee}-\left(1+\delta_{1}-\delta_{2}\right) \omega\left(x_{1}^{\vee}, x_{2}^{\vee}\right)\right) \\
& p_{2, K}^{\vee}\left(x_{1}^{\vee}, x_{2}^{\vee}\right)=\frac{1}{4}\left(1+x_{1}^{\vee}+x_{2}^{\vee}+\left(1-\delta_{1}-\delta_{2}\right) \omega\left(x_{1}^{\vee}, x_{2}^{\vee}\right)\right) \\
& p_{3, K}^{\vee}\left(x_{1}^{\vee}, x_{2}^{\vee}\right)=\frac{1}{4}\left(1-x_{1}^{\vee}+x_{2}^{\vee}-\left(1-\delta_{1}+\delta_{2}\right) \omega\left(x_{1}^{\vee}, x_{2}^{\vee}\right)\right) \\
& p_{4, K}^{\vee}\left(x_{1}^{\vee}, x_{2}^{\vee}\right)=\frac{1}{4}\left(1-x_{1}^{\vee}-x_{2}^{\vee}+\left(1+\delta_{1}+\delta_{2}\right) \omega\left(x_{1}^{\vee}, x_{2}^{\vee}\right)\right)
\end{aligned}
$$


where

$$
\begin{aligned}
\omega\left(x_{1}^{\vee}, x_{2}^{\vee}\right)= & \frac{3 \delta_{1} \delta_{2}}{\left(1-\left(\delta_{1}+\delta_{2}\right)^{2}\right)\left(1-\left(\delta_{1}-\delta_{2}\right)^{2}\right)}\left(1-\left(x_{1}^{\vee}\right)^{2}-\left(x_{2}^{\vee}\right)^{2}\right) \\
& -\frac{\left(1-2 \delta_{1}^{2}-2 \delta_{2}^{2}+\delta_{1}^{4}+7 \delta_{1}^{2} \delta_{2}^{2}+\delta_{2}^{4}-3 \delta_{1}^{2} \delta_{2}^{4}-3 \delta_{1}^{4} \delta_{2}^{2}\right)}{\left(1-\delta_{1}^{2}\right)\left(1-\delta_{2}^{2}\right)\left(1-\left(\delta_{1}+\delta_{2}\right)^{2}\right)\left(1-\left(\delta_{1}-\delta_{2}\right)^{2}\right)} x_{1}^{\vee} x_{2}^{\vee} \\
& +\frac{\delta_{2}\left(1+\delta_{1}^{2}-2 \delta_{2}^{2}-2 \delta_{1}^{4}+\delta_{1}^{2} \delta_{2}^{2}+\delta_{2}^{4}\right)}{\left(1-\delta_{1}^{2}\right)\left(1-\delta_{2}^{2}\right)\left(1-\left(\delta_{1}+\delta_{2}\right)^{2}\right)\left(1-\left(\delta_{1}-\delta_{2}\right)^{2}\right)}\left(x_{1}^{\vee}\right)^{2} x_{2}^{\vee} \\
& +\frac{\delta_{1}\left(1-2 \delta_{1}^{2}+\delta_{2}^{2}+\delta_{1}^{4}+\delta_{1}^{2} \delta_{2}^{2}-2 \delta_{2}^{4}\right)}{\left(1-\delta_{1}^{2}\right)\left(1-\delta_{2}^{2}\right)\left(1-\left(\delta_{1}+\delta_{2}\right)^{2}\right)\left(1-\left(\delta_{1}-\delta_{2}\right)^{2}\right)} x_{1}^{\vee}\left(x_{2}^{\vee}\right)^{2} .
\end{aligned}
$$

\section{Remarks}

- The finite element basis depends on $\delta$.

- if $\delta=0$ (i.e. $K$ is a parallelogram) then $\left(K, P_{K}, S_{K}\right)$ coincides with the classical bilinear finite element.

- $P_{K}=\operatorname{span}\left(1, x_{1}, x_{2}, F_{K}^{\sharp}(\omega)\right)$.

- Numericaly, it is more efficient to calculate the finite element basis by solving a linear system of order eight than to obtain the explicit basis of $P_{K}$.

Proposition 5.2 We assume that there exists $\alpha>0$ such that for each $K \in$ $\mathcal{T}_{h}, \quad\left|\delta_{1}\right|+\left|\delta_{2}\right| \leq 1-\alpha$. Then the assumptions of Propositions 4.1 and 4.2 are satisfied.

Proof The inclusions $P_{1} \subseteq P_{K} \subseteq P_{3}$ are obvious.

Since $\left|\delta_{1}\right|+\left|\delta_{2}\right| \leq 1-\alpha, \frac{1}{|\operatorname{det} R|}$ is bounded, and consequently the $P_{i, K}$ 's are bounded on $K^{2 \#}$. Finally, by construction, the patch test is satisfied.

\subsection{Mixed finite elements}

Let us recall the definition of $B D M_{[k]}$ for $k \geq 1$

$$
\begin{array}{r}
B D M_{[k]}=\left\{\mathbf{w}^{\vee} \mid \mathbf{w}^{\vee}=\mathbf{v}\left(x_{1}^{\vee}, x_{2}^{\vee}\right)+r \operatorname{curl}\left(x_{1}^{\vee k+1} x_{2}^{\vee k}\right)\right. \\
\left.+\operatorname{scurl}\left(x_{1}^{\vee k} x_{2}^{\vee k+1}\right), \mathbf{v}\left(x_{1}^{\vee}, x_{2}^{\vee}\right) \in\left(P_{k}\right)^{2}\right\}
\end{array}
$$

We denote by $B D M_{[1]}^{K}$ the following space:

$$
B D M_{[1]}^{K}=\left\{\mathcal{P}_{K^{\vee}} \circ \mathbf{w}^{\vee} \circ\left(F_{K}^{\sharp}\right)^{-1} ; \mathbf{w}^{\vee} \in B D M_{[1]}\right\}
$$

where $\mathcal{P}_{K^{\vee}}$ is the piola's transform defined by

$$
\mathbf{w}^{\vee} \longrightarrow \frac{1}{\operatorname{det} B_{K}} B_{K} \mathbf{w}^{\vee}
$$

We denote by $B D M K$ the following space: 
We have for any function $\mathbf{w}$ in $P_{0}^{2} \oplus \mathbf{x} P_{0}$

$$
\forall p \in P_{1}, \int_{\gamma_{m}} p \mathbf{w} \cdot \mathbf{n} d \sigma=\frac{1}{\left|\gamma_{m}\right|} \int_{\gamma_{m}} p d \sigma \int_{\gamma_{m}} \mathbf{w} \cdot \mathbf{n} d \sigma .
$$

The result is clear for $\mathbf{w}$ in $P_{0}^{2}$. Moreover it is true for $\mathbf{w}=\mathbf{x}$ since $\mathbf{x} . \mathbf{n}$ remains constant for geometrical reasons.

Let us consider now the following vectorial polynomial space:

$$
\begin{array}{r}
\Psi_{K}=\left\{\mathbf{w} \in B D M_{[1]}^{K} ; \text { for } 1 \leq m \leq 4, \forall p \in P_{1},\right. \\
\left.\int_{\gamma_{m}} p \mathbf{w} \cdot \mathbf{n} d \sigma=\frac{1}{\left|\gamma_{m}\right|} \int_{\gamma_{m}} p d \sigma \int_{\gamma_{m}} \mathbf{w} \cdot \mathbf{n} d \sigma\right\} .
\end{array}
$$

Clearly we have $P_{0}^{2} \oplus \mathbf{x} P_{0} \subseteq \Psi_{K}$. Thus, we have the following result:

Proposition 5.3 For any convex quadrilateral $K$, the triad $\left(K, \Psi_{K}, \Sigma_{K}\right)$ is a Raviart-Thomas finite element.

Proof We proceed in the same way than for Proposition 5.1. For any polynomial w we set :

$$
\widehat{I}_{m}(\mathbf{w})=\int_{\widehat{\gamma}_{m}} \widehat{\mathbf{w}} \cdot \mathbf{n} d \sigma \quad 1 \leq m \leq 4, \quad \widehat{I}_{m}(\mathbf{w})=\int_{\widehat{\gamma}_{m-4}} \sigma \widehat{\mathbf{w}} \cdot \mathbf{n} d \sigma \quad 5 \leq m \leq 8
$$

and

$$
I_{m}^{\vee}(\mathbf{w})=\int_{\gamma_{m}^{\vee}} \mathbf{w}^{\vee} \cdot \mathbf{n} d \sigma \quad 1 \leq m \leq 4, \quad I_{m}^{\vee}(\mathbf{w})=\int_{\gamma_{m-4}^{\vee}} \sigma \mathbf{w}^{\vee} \cdot \mathbf{n} d \sigma \quad 5 \leq m \leq 8 .
$$

Next we introduce the polynomials $r_{j} \in B D M_{[1]}$ satisfying

$$
\widehat{I}_{m}\left(r_{j}\right)=\delta_{m, j}, 1 \leq m, j \leq 8
$$

The $r_{j}$ 's exist since they correspond to the basis of the $B D M_{[1]}$ finite element. Then we consider the square matrix $T$ of order 8 such that $T_{m, j}=I_{m}^{\vee}\left(r_{j}\right)$. The symbolic calculus gives $\operatorname{det} T=\operatorname{det} R$ (where $R$ is the matrix defined in Proposition 5.1. So the proof is achieved.

\section{Remarks}

- if $d=0$ (i.e. $K$ is a parallelogram) then $\left(F_{K}^{\sharp}\right)^{-1}\left(\Psi_{K}\right)=R T_{[0]}^{K}=$ $\left\{\mathbf{q}^{\vee} \circ\left(F_{K}^{\sharp}\right)^{-1} ; \mathbf{q}^{\vee} \in R T_{[0]}\right\}$.

- The finite element $\left(K, \Psi_{K}, \Sigma_{K}\right)$ can be built by using the De Rham diagram $H^{1 \stackrel{\text { curl }}{\longrightarrow}} H(d i v)$ and

$$
\left(F_{K}^{\sharp}\right)^{-1}\left(\Psi_{K}\right)=\operatorname{span}\left(\phi_{1}=\left(\begin{array}{l}
1 \\
0
\end{array}\right), \phi_{2}=\left(\begin{array}{l}
0 \\
1
\end{array}\right), \phi_{3}=\left(\begin{array}{c}
x_{1}^{\vee} \\
x_{2}^{\vee}
\end{array}\right), \phi_{4}=\operatorname{curl}\left(\omega_{K}\right)\right),
$$


with these notations the image by $F_{K}^{\sharp}$ of the basis fonctions of $\boldsymbol{\Psi}_{K}$ are

$$
\begin{aligned}
& \psi_{1, K}^{\vee}\left(x_{1}^{\vee}, x_{2}^{\vee}\right)=\frac{1}{8}\left(2-\delta_{2}\right) \phi_{1}-\frac{1}{8} \delta_{1} \phi_{2}+\frac{1}{8} \phi_{3}+\frac{1}{8}\left(\delta_{1}^{2}-\left(1-\delta_{2}\right)\right)^{2} \phi_{4} \\
& \psi_{2, K}^{\vee}\left(x_{1}^{\vee}, x_{2}^{\vee}\right)=-\frac{1}{8} \delta_{2} \phi_{1}+\frac{1}{8}\left(2-\delta_{1}\right) \phi_{2}+\frac{1}{8} \phi_{3}-\frac{1}{8}\left(\delta_{2}^{2}-\left(1-\delta_{1}\right)\right)^{2} \phi_{4} \\
& \psi_{3, K}^{\vee}\left(x_{1}^{\vee}, x_{2}^{\vee}\right)=\frac{1}{8}\left(2+\delta_{2}\right) \phi_{1}-\frac{1}{8} \delta_{1} \phi_{2}+\frac{1}{8} \phi_{3}+\frac{1}{8}\left(\delta_{1}^{2}-\left(1+\delta_{2}\right)\right)^{2} \phi_{4} \\
& \psi_{4, K}^{\vee}\left(x_{1}^{\vee}, x_{2}^{\vee}\right)=-\frac{1}{8} \delta_{2} \phi_{1}-\frac{1}{8}\left(2+\delta_{1}\right) \phi_{2}+\frac{1}{8} \phi_{3}-\frac{1}{8}\left(\delta_{2}^{2}-\left(1+\delta_{1}\right)\right)^{2} \phi_{4}
\end{aligned}
$$

Proposition 5.4 We assume that there exists $\alpha>0$ such that for each $K \in$ $\mathcal{T}_{h}, \quad\left|\delta_{1}\right|+\left|\delta_{2}\right| \leq 1-\alpha$. Then the assumptions of Propositions 4.4 and 4.5 are satisfied.

Proof The inclusions $P_{0}(K)^{2} \oplus \mathbf{x} P_{0}(K) \subset \Psi_{K} \subset P_{2}(K) \times P_{2}(K)$ are obvious.

Since $\left|\delta_{1}\right|+\left|\delta_{2}\right| \leq 1-\alpha, \frac{1}{|\operatorname{det} T|}$ is bounded, and consequently the $\psi_{i, K}$ 's are bounded on $K^{2 \#}$. Finally, by construction, the patch test is satisfied.

\section{Numerical tests}

We take $\Omega=] 0,1[\times] 0,1\left[\right.$ and the exact solution is $u\left(x_{1}, x_{2}\right)=\sin \left(\pi x_{1}\right) \sin \left(\pi x_{2}\right)$. We considere two types of mesh. They are composed of two patterns and their shapes are the same for each mesh used, see Figure 3. The first mesh is a mesh in chevron given in [9] and the second is a mesh in honeycomb.

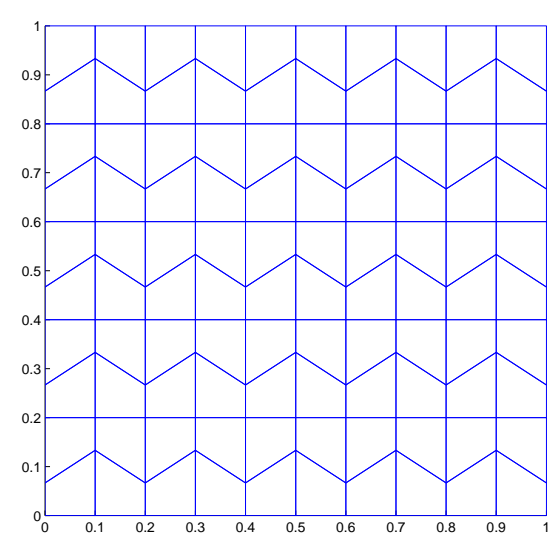

Mesh 1 (Chevron mesh)

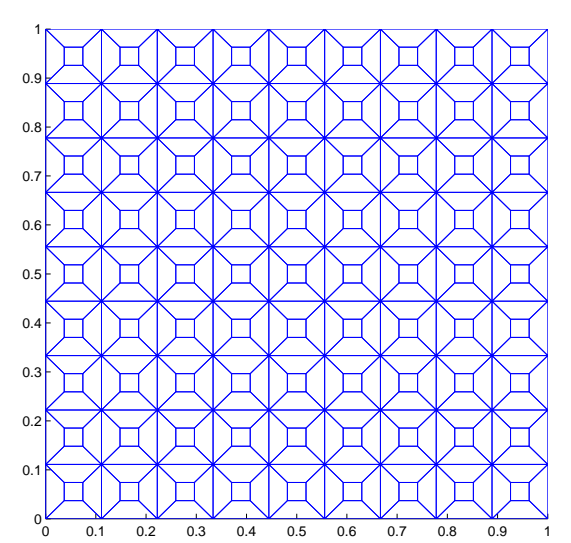

Mesh 2 (Honeycomb mesh)

Figure 3. meshes 1 and 2

\subsection{Lagrange finite element}

In the first test, we take $P_{K}=Q_{1}^{K}$ and as expected the method does not converge on (deformed) quadrilateral meshes but converges on meshes based on squares or parallelograms, see Figure 4 . In the second test, $P_{K}$ is given by (20). For the two meshes proposed, we obtain the expected order of convergence, see Figure 5. 

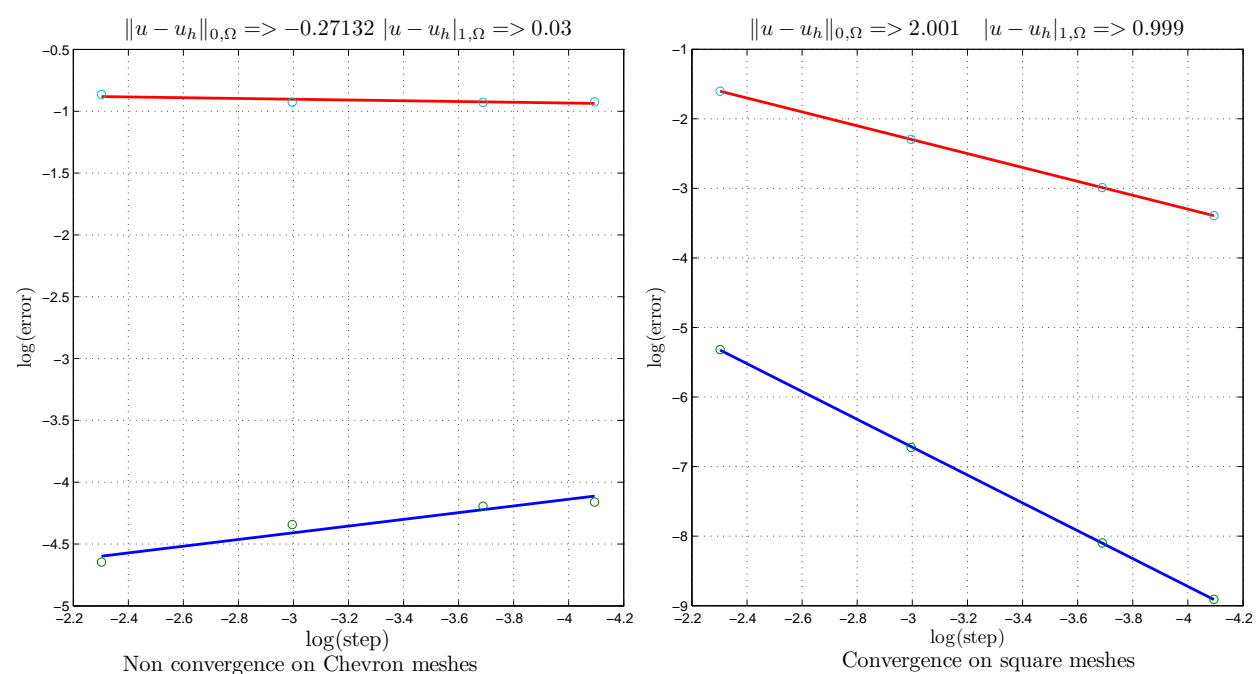

Figure 4. Convergence curves when $P_{K}=Q_{1}^{K}$
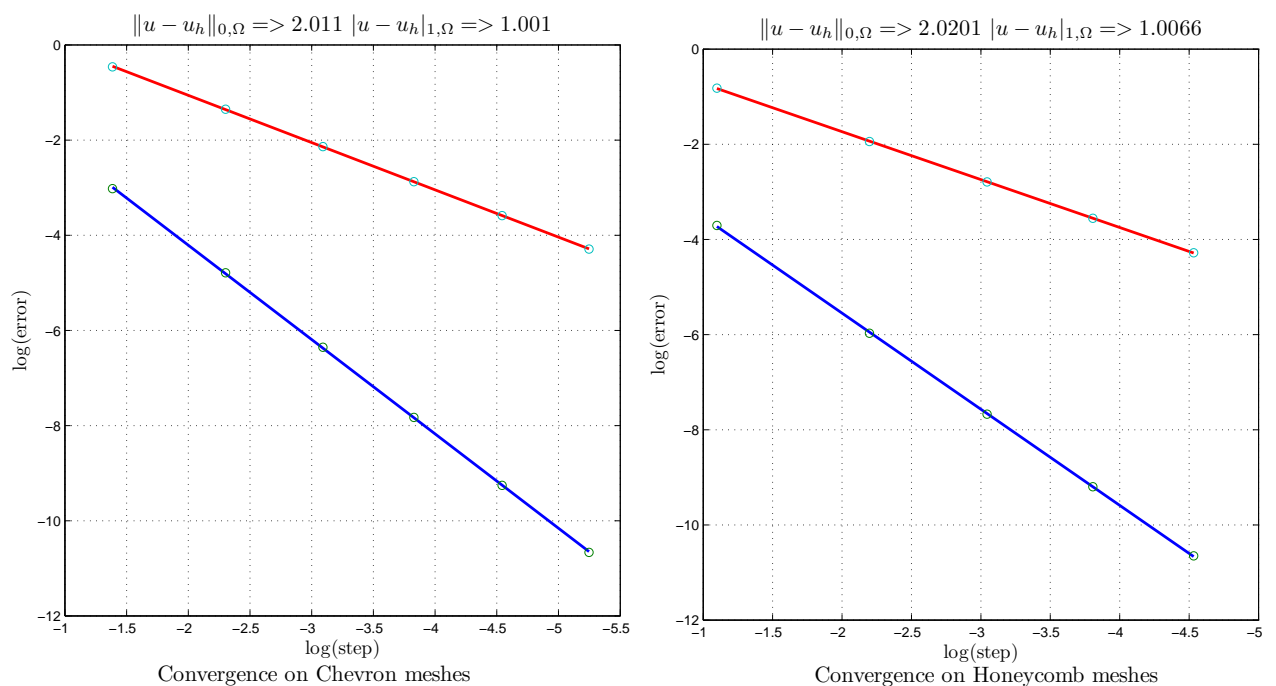

Figure 5. Convergence curves when $P_{K}$ is given by (20)

\subsection{Mixed finite element}

The classical mixed finite element method (2D or $3 \mathrm{D}$ case) ([5],[3]) corresponds to the problem (16),(17) by substituting $L_{h}$ for a conform subspace of $H(d i v, \Omega)$ i.e.

$$
\left\{\mathbf{q}_{h} \in H(\operatorname{div}, \Omega) ;\left.\widehat{\mathbf{q}}_{h}\right|_{\widehat{K}} \in R T_{[0]}(\widehat{K}) \text { for each } K \in \mathcal{T}_{h}\right\},
$$

where $\widehat{\mathbf{q}}_{h}$ is defined by the non linear Piola transform :

$$
\mathbf{q}_{h}=\frac{1}{\operatorname{det} J_{F}} J_{F} \widehat{\mathbf{q}}_{h}
$$

( $J_{F}$ being the jacobian of the transformation of $K$ into $\left.\widehat{K}\right)$.

As mentioned in the introduction, the obtained approximation $\left(u_{h}, \mathbf{q}_{h}\right)$ does not converge in $L^{2}(\Omega) \times H($ div,$\Omega)([9])$. On general quadrilaterals, $u_{h}$ and $\mathbf{p}_{h}$ converge 
in $L^{2}(\Omega)$ but $\mathbf{p}_{h}$ does not converge in $H(d i v, \Omega)$. In the $3 \mathrm{D}$ case on general hexahedra, the situation is worse since $u_{h}$ and $\mathbf{p}_{h}$ do not converge (see Figure 6).

The rates of convergence on Figure 7 correspond to $\Psi_{K}$ given by (21) and as
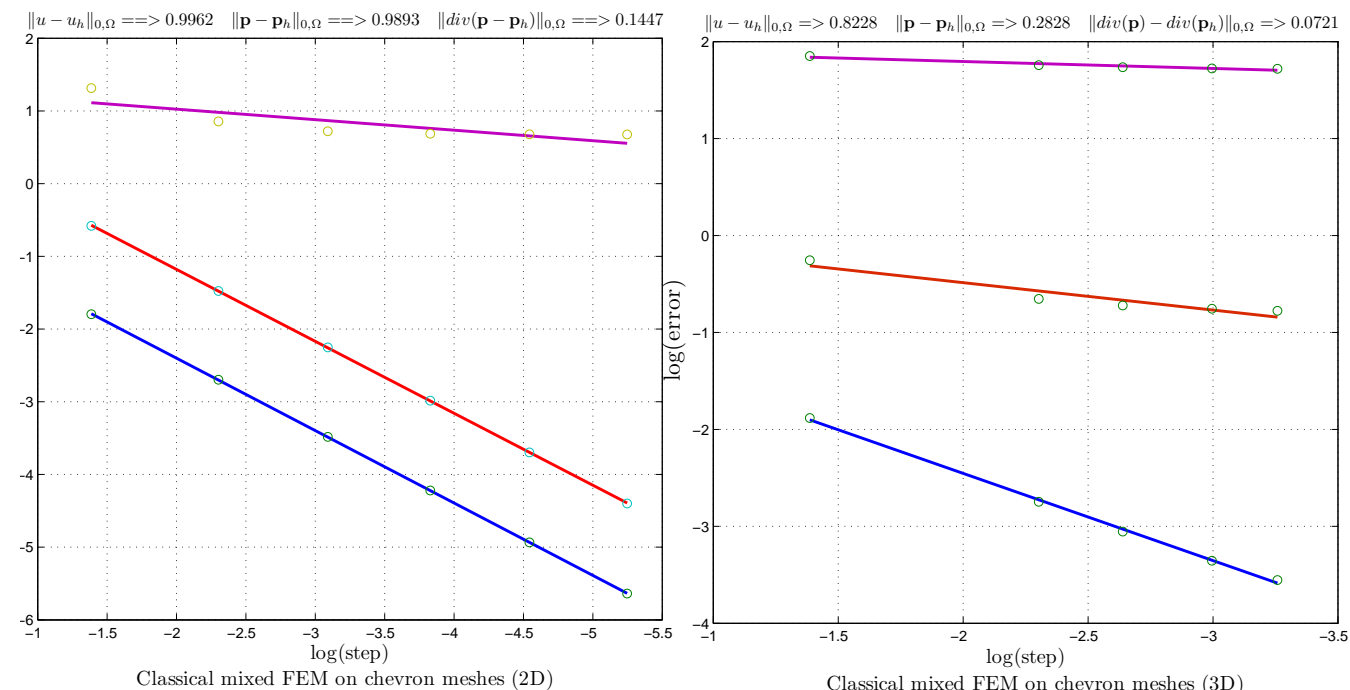

Figure 6. Rates of convergence of classical mixed finite element

expected $u_{h}$ (resp. $\mathbf{p}_{\mathbf{h}}$ ) converges in $L^{2}$ (resp. Hdiv) with the order 1 .
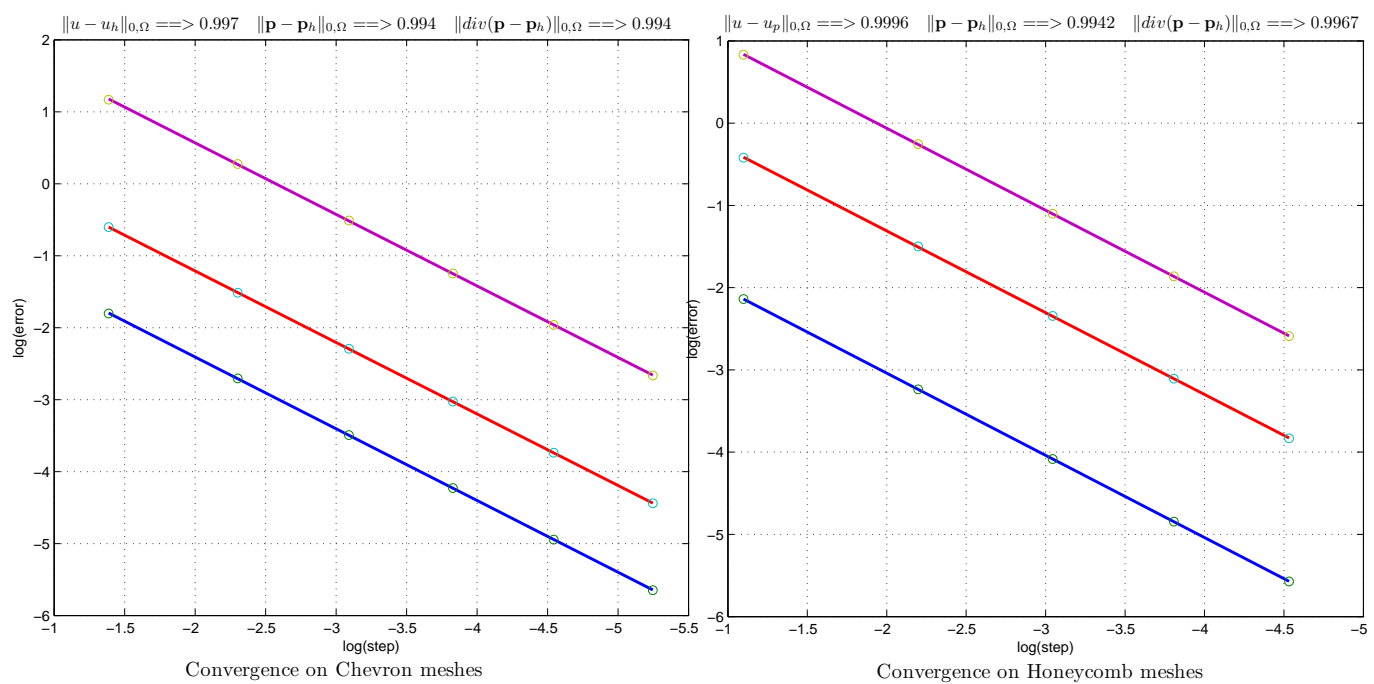

Figure 7. Rates of convergence when $\Psi_{K}$ is given by (21)

\section{Conclusion}

One of the motivations of this work refers to the loss of convergence problem when using classical mixed finite elements on quadrilaterals and hexahedra (see for instance [9], [4]). The pseudo-conforming finite elements are a good answer to this problem. The process discribed can be extended to the 3D case, pseudo-conforming 
Lagrange finite elements on hexaedra are presented in ([10]), and the case of mixed finite elements will be treated in a paper to come. Note that another way for obtaining polynomial basis functions is to cut the quadrilaterals into triangles (or hexahedra into tetrahedra) and work with macro-elements ([11], [12], [4]).

\section{References}

[1] P.. G. Ciarlet, The Finite Element Method for Elliptic Problems Classics in Applied Mathematics, Vol.40, SIAM, Philadelphia, 2002. First edition published by North-Holland, Amsterdam, 1978.

[2] P.. G. Ciarlet, Basic error estimates for elliptic problems, in "Handbook of Numerical Analysis, Vol.II" (eds. P.G. Ciarlet and J.L. Lions), Elsevier, (1991), 17-351.

[3] J. E. Roberts, J.-M. Thomas Mixed and hybrid methods. In Handbook of Numerical Analysis, VOL.II, Finite Element Methods (Part 1), Elsevier Science Publishers B.V. (North-Holland), Amsterdam (1991) (1977) 523-639,.

[4] A. Sboui, J. E. Roberts And J. Jaffré, A Composite Mixed Finite element for general hexahedral grids for Darcy flow calculations, Preprint INRIA, submittted to SIAM

[5] F. Brezzi, M. Fortin, Mixed and Hybrid Finite Element Methods, Springer-Verlag, Berlin, 1991.

[6] G. Strang And G.J. Fix, An Analysis of the Finite Element Method, Prentice-Hall,Englewood Cliffs, NJ, 1973

[7] D. N. Arnold, D. Boffi, R.S. FAlK, Approximation by quadrilateral finite elements, Maths of Comp., 239 (2002), 909 - 922.

[8] S. C. Brenner L. R. Scott, The Mathematical Theory of Finite Element Methods Series: Texts in Applied Mathematics , Vol. 15 2nd ed., 2002

[9] D. N. Arnold, D. Boffi, R. S. FAlk, Quadrilateral H(div) finite elements, SIAM J. Numerical Analysis, 42 (2005) 2529-2451.

[10] E. Dubach, R. Luce, J.M. Thomas, Peuso-conform Lagrange Finite Elments on quadrilaterals and Hexahedra Rapport de recherche RR-6572 (2008) [inria-00293546 version 1]

[11] Y. Kuznetsov, S. RePin, New mixed finite element method on polygonal and polyhedral meshes, Russ. J. Numer. Anal. Math. Model. 18 (3) (2003) 261-278.

[12] Y. Kuznetsov, S. Repin, Convergence analysis and error estimates for mixed finite element method on distorted meshes, J. Numer. Math. 13 (1) (2005) 33-51. 